\title{
La economía del crecimiento impulsado por la demanda. Teoría y evidencia respecto del Brasil
}

\author{
José Luis Oreiro, Luciano Nakabashi, Guilherme Jonas \\ Costa da Silva y Gustavo José Guimarães e Souza
}

RESUMEN

Se describe la teoría del crecimiento impulsado por la demanda y se aporta evidencia de la existencia de un modelo semejante en la economía brasileña. A partir de la metodología desarrollada por Atesoglu (2002), se realizaron pruebas econométricas para corroborar la hipótesis de que la economía brasileña se basa en el crecimiento promovido por la demanda. Los resultados indican que prácticamente el $85 \%$ de la tasa de crecimiento del PIB real en el período 1990-2005 se explica por variables de la demanda, principalmente las exportaciones y el consumo público. Dado que la actual crisis fiscal elimina la posibilidad de expansión fiscal, la única opción para el Brasil es adoptar un modelo de crecimiento impulsado por las exportaciones. En este artículo se demuestra también que el mantenimiento de un tipo de cambio real (TCR) devaluado es un factor importante para el incremento de las exportaciones en países en desarrollo como el Brasil.

PALABRAS CLAVE

CLASIFICACIÓN JEL

AUTORES
Crecimiento económico, modelos de desarrollo, oferta y demanda, tipos de cambio, exportaciones, modelos econométricos, macroeconomía, Brasil

$\mathrm{E} 12, \mathrm{C} 1, \mathrm{~F} 43$

José Luís Oreiro. Profesor adjunto de Economía de la Universidad de Brasilia, Investigador del Consejo Nacional de Desarrollo Científico y Tecnológico. joreiro@unb.br

Luciano Nakabashi. Profesor adjunto de Economía de la Universidad de São Paulo, FEA-RP/usp, Investigador del Consejo Nacional de Desarrollo Científico y Tecnológico. luciano.nakabashi@gmail.com

Guilherme Jonas Costa da Silva. Profesor adjunto de Economía de la Universidad Federal de Uberlândia (UFU). guilhermejonas@yahoo.com.br

Gustavo José Guimarães e Souza. Estudiante de doctorado en la Universidad de Brasilia, Profesor de la Universidad Católica de Brasilia.gustavojgs@gmail.com 


\section{I}

\section{Introducción}

Durante los últimos 25 años la economía brasileña creció a una tasa media del $2,6 \%$ anual, ritmo considerablemente inferior al registrado en el período 1950-1980 y a las tasas medias de crecimiento correspondientes a otras economías emergentes, tales como la Federación de Rusia, la India y China. Con un aumento de la población del Brasil de prácticamente un 1,5\% anual, el producto interno bruto (PIB) per cápita se eleva a cerca de un $1 \%$ anual. A este ritmo, deberían transcurrir casi 70 años para que el PIB per cápita alcanzara los niveles que actualmente ostentan España o Portugal. En este sentido, la situación de la economía brasileña es hoy de un cuasi estancamiento.

A finales de la década de 1980 y principios de los años noventa, se consideraba que dicha situación obedecía a la alta inflación que dominaba constantemente la economía brasileña. En marzo de 1990, que fue el último mes del mandato del presidente Sarney, ello terminó convirtiéndose en hiperinflación, registrándose un alza del $72 \%$ mensual en los precios. Con la exitosa implementación del Plan Real en la primera presidencia de Fernando Henrique Cardoso se logró reducir las tasas de inflación anual a menos del $10 \%$. Este proceso implicó anclar la inflación al tipo de cambio bajo el sistema de paridad cambiaria móvil, que se puso en práctica desde 1995 hasta 1998.

Sin embargo, tras la estabilización no se produjo una aceleración sostenida del producto. El crecimiento más rápido registrado en los dos primeros años del Plan Real, con tasas medias cercanas al 5\% anual, llegó a su fin debido al contagio de las crisis externas de México, Asia oriental y la Federación de Rusia.

A principios de 1999, tras una enorme pérdida de reservas internacionales generada por la repentina interrupción de la llegada de capitales a la economía brasileña, a la vez que se evaporaba la confianza en la sostenibilidad del régimen cambiario del país, las autoridades monetarias del Brasil adoptaron un régimen cambiario flexible.

En 1999 se completó el nuevo modelo macroeconómico con la adopción de objetivos directos de inflación,

$\square$ Los autores agradecen los útiles comentarios de Luiz Carlos Bresser-Pereira, Malcon Sawyer, Giusepe Fontana, José Gabriel Porcile Meirelles, Marco Crocco, Frederico Jayme Gonzaga y de un evaluador anónimo de la Revista CEPAL. potenciados por una política fiscal destinada a generar importantes niveles de superávit primario tendientes a evitar que se disparara la relación entre la deuda pública y el PIB.

Con el nuevo modelo macroeconómico se sentaron las bases para reducir marcadamente las tasas de interés real — de casi el 25\% anual en el período 1994-1998 a cerca de un 10\% en el período 1999-2005- y devaluar el tipo de cambio real, una medida clave para eliminar los déficits registrados en la cuenta corriente en el período 1994-1998, que treparon a casi un 4\% del PIB. Por otra parte, el hecho de contar con una política fiscal que generaba relevantes superávits primarios hizo posible aminorar la relación entre la deuda pública y el PIB del 63\% alcanzado en 2002 a su nivel actual de aproximadamente $45 \%$.

A pesar de las disminuidas tasas de interés real, la menor fragilidad externa y la estabilización de la deuda pública, el crecimiento de la economía brasileña sigue siendo muy débil. En el período 1999-2005, el crecimiento anual medio fue apenas de un 2,3\%, en comparación con un 3,22\% del período 1994-1998.

En este contexto, el problema principal radica en cómo lograr que la tasa de crecimiento de la economía brasileña aumente en forma sostenida.

Existen dos respuestas a este interrogante. En la primera, basada en modelos neoclásicos de crecimiento y la metodología para su análisis, se plantea que el motivo del débil dinamismo de la economía brasileña en los últimos 25 años ha de buscarse en la oferta de la economía. Más específicamente, los motivos de la baja tasa de incremento del PIB fueron el escaso nivel de ahorro interno - debido al aporte negativo del sector público y a los escasos incentivos al ahorro privadoy la falta de dinamismo tecnológico reflejada en una bajísima tasa de crecimiento de la productividad total de los factores. Desde esta perspectiva, el incremento sostenido de dicha tasa requeriría de la aplicación de reformas en el sistema de la seguridad social, a objeto de aumentar el ahorro del sector público con el apoyo de una economía más abierta que permita estimular una mayor productividad de las empresas brasileñas.

El segundo enfoque para abordar la cuestión se basa en la idea de que el modelo macroeconómico adoptado en el Brasil en la última década ha menoscabado la demanda 
agregada y está obstaculizando la tasa de crecimiento real del PIB. Ello se debe a que al combinarse tasas de interés real —que aún son elevadas — con la generación de importantes superávits primarios — que en los últimos años han ido en aumento- se está deprimiendo la demanda. De acuerdo con esta óptica, la solución para el cuasi estancamiento que sufre la economía del Brasil consistiría en reemplazar el modelo macroeconómico actual, basado en objetivos directos de inflación, con tasas cambiarias flexibles y la generación de superávits primarios.

Bajo la premisa de que ambas posturas son incorrectas, en este artículo se adopta un criterio keynesiano en que los factores determinantes del crecimiento a largo plazo han de buscarse en la demanda, más que en la oferta de la economía. No obstante, se rechaza la ingenua postura keynesiana según la cual cualquier política que acreciente la demanda agregada es capaz de estimular el crecimiento. La crisis fiscal imperante en el Brasil impone claras restricciones a las políticas fundadas en el aumento del consumo público. La expansión sostenida de la economía brasileña requiere la adopción de un nuevo modelo de crecimiento donde las exportaciones impulsen la demanda agregada y funcionen, por ende, como motor del dinamismo a largo plazo. Sin embargo, para adoptar tal modelo es necesario contar con un régimen cambiario capaz de mantener subvalorado el tipo de cambio real (TCR).
Este artículo se divide en cinco secciones, incluida la Introducción. En la sección II se describe la teoría del crecimiento impulsado por la demanda, en que la tasa de incremento a largo plazo del PIB real es una media ponderada de las tasas de variación del consumo público y de las exportaciones. En la sección III, sobre la base de la metodología desarrollada por Atesoglu (2002), se exponen las pruebas econométricas de que la economía brasileña es un modelo de crecimiento impulsado por la demanda. Los resultados de dichas pruebas demostraron que prácticamente el $85 \%$ del aumento del PIB en el período 1990-2005 se explica por variables de la demanda. Por otra parte, las pruebas basadas en la metodología desarrollada por Ledesma y Thirlwall (2002) demuestran que la tasa natural de crecimiento de la economía del Brasil es endógena y considerablemente superior en períodos de auge. Estos resultados permiten apreciar que no existen restricciones desde la oferta que funcionen como óbice frente a un incremento sostenido de la tasa de crecimiento de la economía brasileña. En la sección IV se ofrece un análisis empírico de la relación entre el tipo de cambio real y la elasticidad-ingreso de las exportaciones, a objeto de demostrar que para poner en práctica un modelo de crecimiento impulsado por las exportaciones es necesario fijar el TCR en niveles competitivos. En la sección V se resumen las conclusiones.

\section{II}

\section{La teoría del crecimiento impulsado por la demanda: la postura keynesiana}

\section{La endogeneidad a largo plazo de la oferta de los factores de producción}

Los modelos neoclásicos de crecimiento se basan en el supuesto de que el límite fundamental a largo plazo a la expansión está dado por la oferta de los factores de producción. La demanda agregada solo reviste importancia para los efectos de determinar el grado de utilización de la capacidad productiva, aunque no tiene influencia directa en la tasa de crecimiento de dicha capacidad. En el largo plazo, se da por sentado que se cumple la ley de Say: la oferta genera su propia demanda.

Pero, ¿la oferta de los factores de producción es verdaderamente independiente de la demanda? Planteado originalmente por Kaldor (1988), este interrogante dio lugar a la teoría del crecimiento impulsado por la demanda, sobre la base de la idea de que en una economía capitalista moderna los medios de producción son, en sí mismos, bienes producidos dentro del sistema. La "oferta" de los medios de producción jamás debería darse por supuesta ni considerarse independiente de su demanda. En este marco teórico, el problema económico principal no radica en distribuir una cantidad dada de recursos entre usos alternativos, sino en determinar la tasa a la que se crearán esos recursos.

Como punto de partida para entender la endogeneidad a largo plazo de los factores de producción se puede comenzar por la oferta de capital. El volumen del 
capital existente en un momento dado —o la capacidad productiva existente en la economía - es el resultado de las decisiones de inversión tomadas en el pasado. De ello se concluye que el volumen de la acumulación de capital no se determina en forma natural, sino que depende de la tasa a la que los empresarios deseen aumentarlo.

Esto significa que las decisiones de inversión son los factores que más influyen en la formación del acervo de capital. A su vez, las inversiones están determinadas por dos grupos de variables, a saber: i) el costo de oportunidad del capital (determinado principalmente por la tasa de interés a corto plazo que fije el banco central), y ii) las expectativas de aumento de las ventas y la producción. En este contexto, si los empresarios prevén un incremento fuerte y sostenido de la demanda de los bienes que producen — tal como cabría esperar en una economía con una tasa de crecimiento constantemente elevada—, realizarán importantes gastos de inversión.

En otras palabras, la inversión es una variable endógena que acompaña al crecimiento esperado de la demanda agregada cuando se cumple una condición fundamental: la tasa esperada de rendimiento del capital debe superar el costo del capital. Ante este supuesto no cabe considerar que la "oferta de capital" sea una restricción al crecimiento a largo plazo.

Si bien en el corto y el mediano plazo la producción no puede superar la máxima capacidad productiva de la economía, en el largo plazo debe acrecentarse la capacidad productiva - por medio de gastos de inversión - de modo de satisfacer el incremento de la demanda agregada.

El segundo punto de atención está dado por la "oferta de mano de obra", que en esta teoría tampoco se considera como restricción al crecimiento de la producción a largo plazo.

Por una parte, la cantidad de horas trabajadas puede aumentarse fácilmente a fin de elevar el nivel producido.

Por otra, la tasa de participación laboral - la relación entre la fuerza de trabajo y la población en edad de trabajar- puede crecer en respuesta a un marcado incremento en la demanda de mano de obra (Thirlwall, 2002, pág. 86). De hecho, durante los períodos de auge, sube el costo de oportunidad del ocio, lo que sirve de estímulo para un robusto aumento en la tasa de participación. Así, la fuerza de trabajo puede engrosarse con mayor rapidez durante los períodos de auge debido a la incorporación de trabajadores que responden a los incentivos que genera un mercado laboral en auge.

Cabe destacar que la población y la fuerza de trabajo no son referencias para la economía en su conjunto. La falta de mano de obra -incluso de trabajadores calificados- puede resolverse mediante la inmigración de otros países. Por ejemplo, hay países como Alemania y Francia que lograron sostener altos niveles de crecimiento en las décadas de 1950 y 1960 mediante el empleo de trabajadores provenientes de la periferia europea (España, Portugal, Grecia, Turquía y el sur de Italia).

Por último, vale la pena analizar si la tasa de progreso tecnológico opera como una restricción al crecimiento a largo plazo, el cual quedará limitado por el ritmo al que se amplíe el conocimiento de las tecnologías de la información y la comunicación (TIC) si el progreso tecnológico es exógeno al sistema económico; sin embargo, se observa el supuesto contrario.

En primer lugar, uno de los principales factores que repercuten en el ritmo de innovación de las empresas es la tasa de acumulación de capital, ya que parte importante de las innovaciones tecnológicas está incorporada en las nuevas máquinas y equipos ${ }^{1}$.

En segundo lugar, incluso la pequeña parte del progreso tecnológico que no está incorporada está determinada por economías dinámicas de escala, como el aprendizaje práctico. Por ende, existe una relación estructural entre la tasa de crecimiento de la productividad del trabajo y la tasa de crecimiento de la producción, que se conoce con el nombre de "Ley de Kaldor-Verdoon"2, 3. En este

\footnotetext{
${ }^{1}$ Se trata de una idea expresada originalmente por Kaldor (1957) mediante la "función de progreso técnico", en que se plantea la existencia de una relación estructural entre la tasa de crecimiento de la producción por trabajador y la tasa de crecimiento del capital por trabajador. Según Kaldor, es imposible aislar el incremento de la productividad del trabajo generado por la incorporación de nuevas tecnologías de aquel proveniente de un aumento en el capital por trabajador. Ello se debe a que prácticamente todas las innovaciones tecnológicas que elevan la productividad del trabajo requieren de mayor capital por trabajador, dado que dichas innovaciones están incorporadas en las nuevas máquinas y los nuevos equipos.

2 En McCombie y De Ridder (1984) podemos encontrar evidencia econométrica de la validez de la "Ley de Kaldor-Verdoon" para los Estados Unidos de América.

${ }^{3}$ Ledesma (2002) estima un modelo de crecimiento impulsado por la demanda para 17 países de la Organización de Cooperación y Desarrollo Económicos (OCDE) en el período 1965-1994: Alemania, Australia, Austria, Bélgica, el Canadá, Dinamarca, España, los Estados Unidos de América, Finlandia, Francia, Italia, el Japón, Noruega, los Países Bajos, Portugal, el Reino Unido de Gran Bretaña e Irlanda del Norte y Suecia. A partir de esta evidencia econométrica se puede identificar una relación estructural entre la tasa de crecimiento de la productividad del trabajo y un conjunto de otras variables, en particular la tasa de crecimiento de la producción. La ecuación estructural estimada es la siguiente:
}

$r=-0,015+0,642 y+0,0002(I / O)+0,617 K+0,021 G A P$

donde $r$ es la tasa de crecimiento de la productividad del trabajo; $y$ es la tasa de crecimiento de la producción real; $(I / O)$ es la inversión, expresada como proporción del PIB real; $K$ es un índice de innovación tecnológica, y GAP es una estimación de la brecha tecnológica. 
marco, un incremento en la demanda agregada provocará un aumento más rápido de la productividad del trabajo, puesto que el crecimiento de la producción se acelerará tras un acrecentamiento más pronunciado de la demanda.

Desde este punto de vista, no es posible concebir una producción potencial o a pleno empleo a largo plazo, ya que tanto la oferta de los factores de producción como la tasa de progreso tecnológico están determinadas por la demanda. El "pleno empleo" es básicamente un concepto a corto plazo que pasa por alto el carácter endógeno de la "tasa natural de crecimiento" a largo plazo.

\section{Los determinantes del crecimiento a largo plazo}

Si no corresponde considerar que los factores de producción conllevan una restricción al crecimiento a largo plazo, ¿cuáles son entonces los determinantes de dicho crecimiento? Desde la perspectiva keynesiana, el factor que más incide en el crecimiento económico es la demanda agregada. Las empresas elevan los niveles de producción en respuesta a un incremento de la demanda agregada, siempre que se encuentren reunidas dos condiciones: i) que los márgenes de ganancia resulten suficientemente altos como para permitir que los empresarios obtengan la tasa de rendimiento deseada, y ii) que la tasa de beneficio efectiva sea superior al costo del capital. $\mathrm{Si}$ se cumplen las dos condiciones, la tasa de crecimiento de la producción real estará entonces determinada por la tasa de crecimiento de la "demanda autónoma", es decir, de aquella porción de la demanda agregada que es independiente del nivel de producción y de ingresos, de las variaciones que en ellos se producen, o de ambos.

Cuando se trata de economías abiertas, la demanda autónoma presenta dos componentes: las exportaciones y el gasto de consumo público (Park M.S., 2000). La inversión no es un componente de la demanda autónoma, ya que las decisiones de invertir en bienes de capital están determinadas, básicamente, por las expectativas de crecimiento futuro de la producción y de las ventas que albergan los empresarios, según el principio de aceleración relativo a la teoría de la inversión (Harrod, 1939). En otras palabras, la inversión no constituye una variable exógena en el proceso de producción, ya que efectivamente la impulsa el crecimiento de la producción. Por ende, la tasa de crecimiento a largo plazo de la producción real constituye una media ponderada de las tasas de crecimiento de las exportaciones y del gasto de consumo público.

Para una economía abierta y pequeña, que no cuenta con su propia moneda convertible, el incremento de las exportaciones es la variable exógena en el proceso de crecimiento. Si el consumo público aumenta a mayor velocidad que las exportaciones, entonces el crecimiento de la producción y de los ingresos reales superará al de las exportaciones. En el supuesto de que la elasticidadingreso de las importaciones es mayor que 1 -como suele suceder en las economías abiertas-, las importaciones crecerán con mayor velocidad que las exportaciones y ello generará un déficit comercial cada vez más agudo (si los términos comerciales se mantienen constantes) que resultará imposible sostener en el largo plazo ${ }^{4}$.

La tasa de crecimiento de las exportaciones se calcula como producto de la elasticidad-ingreso de las exportaciones $(\varepsilon)$ y la tasa de variación del ingreso mundial ( $z$ ). Así, en el modelo de crecimiento impulsado por la demanda la tasa de expansión a largo plazo de la producción real $\left(g^{*}\right)$ está dada por:

$$
g^{*}=\varepsilon z
$$

Es decir, la tasa de crecimiento del producto real es igual al producto entre la elasticidad-ingreso de las exportaciones y la tasa de variación del ingreso mundial.

\footnotetext{
${ }^{4}$ Cabe advertir que el crecimiento de las exportaciones superior al aumento del consumo público no es condición suficiente para que se produzca un proceso de crecimiento sostenible en el largo plazo; también es necesario que la balanza de pagos esté en equilibrio. Para las economías abiertas con nula movilidad de capital, ello significa que la tasa de crecimiento a largo plazo será igual a la relación entre la elasticidad-ingreso de las exportaciones y la elasticidad-ingreso de las importaciones, relación que se multiplica por la tasa de crecimiento de los ingresos mundiales, lo que se conoce con el nombre de "Ley de Thirlwall" (Thirlwall, 1997). La incorporación de flujos de capital no altera sustancialmente la tasa de crecimiento del equilibrio a largo plazo (McCombie y Roberts, 2002, págs. 95-96). Este artículo no se refiere a las restricciones que supone la balanza de pagos para el crecimiento económico brasileño, sino que con él se pretende demostrar para el Brasil la existencia de un modelo de crecimiento impulsado por la demanda. Por ende, en las pruebas econométricas no se utilizará la "Ley de Thirlwall".
} 


\section{III \\ ¿Crecimiento impulsado por la demanda en el Brasil? Algunas pruebas empíricas}

En esta sección se exponen pruebas econométricas de que el crecimiento en la economía brasileña es impulsado por la demanda agregada. En primer lugar, ello permite apreciar que a ciertas variables de la demanda agregada les cabe un papel clave a la hora de explicar la expansión que registró la economía del Brasil en el período 1991-20055. En especial, las exportaciones y el consumo público corriente constituyen variables exógenas en el dinamismo a largo plazo, lo que corrobora el modelo de crecimiento impulsado por la demanda descrito en la sección II. También se demuestra que la tasa natural de expansión de la economía brasileña es endógena y está determinada por la dinámica de la tasa corriente de crecimiento impulsado por la demanda agregada. Esto significa que las condiciones de la oferta no imponen una restricción ineludible al avance económico. Las estimaciones practicadas para los fines de este artículo (en función de datos trimestrales sobre el desempleo y el crecimiento registrados por la economía brasileña en el período 1980-2002) permiten ver que la tasa natural de expansión anual puede variar entre un $5,2 \%$ y un $8 \%$ en períodos de auge.

\section{Contraste de la hipótesis del crecimiento im- pulsado por la demanda}

En esta subsección se utiliza la metodología de Atesoglu (2002) para contrastar la hipótesis de que en la economía brasileña el crecimiento está impulsado por la demanda agregada. Ello implica medir la relación entre el PIB real (Y) y las siguientes variables: nivel real de exportaciones (X); nivel real de inversión (I) ${ }^{6}$; consumo público real (G); y oferta monetaria real (M2 deflactado) ${ }^{7}$.

\footnotetext{
${ }^{5}$ El Instituto Brasileño de Geografía y Estadística (IBGE) reprodujo los cálculos del PIB correspondientes al período 1995-2006. Como la serie analizada es trimestral y el período de análisis de este estudio se extiende desde 1991 hasta 2005, los datos utilizados en las estimaciones son los obtenidos por aplicación de la antigua metodología del IBGE. ${ }^{6}$ Pública y privada.

${ }^{7}$ Es preciso explicar la razón por la que se utiliza una variable de oferta monetaria en lugar de una tasa de interés a largo plazo como sustitutiva de los efectos de la política monetaria en el crecimiento a largo plazo del Brasil. En primer lugar, la aplicación de una política monetaria de fijación del tipo de interés a corto plazo no se inició
}

Los datos correspondientes al PIB real, las exportaciones reales, las inversiones reales y el consumo público real se tomaron del Sistema de Cuentas Nacionales del Instituto Brasileño de Geografía y Estadística (IBGE/SCN). La serie sobre la oferta monetaria se tomó del Banco Central del Brasil (вСв). Todas las series fueron deflactadas por el Índice General de Precios-Disponibilidades Internas (IGP-DI) calculado por la Fundación Getulio Vargas. Todas las variables se transformaron a fin de establecer sus valores de 1991, como año base, en 100 $(1991=100)$, y se aplicaron a estas tasas logaritmos naturales ${ }^{8}$. Como resultado, los coeficientes calculados representan las elasticidades entre las variables en estudio. El período en estudio comprende 60 trimestres, desde el primer trimestre de 1991 hasta el último de 2005.

Para buscar tendencias estocásticas en las variables se aplicaron las siguientes pruebas de raíz unitaria: prueba de Dickey-Fuller aumentada (ADF), prueba de Phillips-Perron (PP) y Dickey-Fuller ajustada por tendencia (DF-GLS), junto con la prueba estacionaria de KPSS, propuesta por Kwiatkowski, Phillips, Schmidt y Shin (1992) $)^{9}$. La decisión de incluir o excluir la

sino en 1999, tras el establecimiento del modelo de objetivos directos de inflación. Con anterioridad a 1999, el Banco Central del Brasil utilizaba otros objetivos operativos para la política monetaria, como el crecimiento de la oferta monetaria (1994-1995) y el tipo de cambio nominal (1996-1998). En segundo lugar, el Brasil no cuenta con un "mercado" de tasas de interés a largo plazo debido a que los títulos del gobierno (Letras Financieras del Tesoro) están indexados en función de la tasa de interés a corto plazo. Esto elimina la posibilidad de que se produzcan pérdidas de capital a raíz de un incremento de la tasa de interés a corto plazo, lo que significa que la "duración" (o sea, la sensibilidad del precio de los bonos a los cambios de la tasa de interés) de los títulos del gobierno es 0 , y esto los convierte en sustitutos perfectos de las reservas bancarias. Esta característica institucional específica de la economía brasileña genera la "contaminación" de la política monetaria por parte del endeudamiento público, lo que da lugar a una "curva de rendimiento horizontal"; en otras palabras, se produce una situación en la que deudas con diferentes vencimientos tienen la misma tasa de interés, es decir, la tasa de interés sobre los préstamos del mercado interbancario (véase Barbosa, 2006).

${ }^{8} \mathrm{La}$ colocación de una $\mathrm{L}$ antes del nombre de cada variable indica su forma logarítmica, mientras que las letras DL denotan la primera diferencia de los logaritmos.

${ }^{9}$ De acuerdo con Maddala (2001), se utilizó el análisis confirmatorio y se adoptó un nivel de significación del $10 \%$. Para el caso de resultados encontrados se dio preferencia a la prueba de raíz unitaria. 
constante o tendencia, o ambas, además de la cantidad de rezagos para cada serie, se tomó mediante la aplicación del criterio de información de Schwarz (SC) y el criterio de Newey-West (NW), con el respaldo de la significación estadística de los parámetros estimados y las pruebas de diagnóstico habituales, siempre comenzando con el modelo general para pasar luego al particular (rezago inicial $=10$ ). Los resultados, que se exponen en el cuadro 1, demuestran que todas las variables son integradas de orden 1, o I(1), y por consiguiente, no son estacionarias.

Al ser todas las series I(1), no se producen problemas de correlación espuria entre las variables dependientes e independientes en los resultados; para estimar la regresión se utilizan variables expresadas como primeras diferencias, tal como se muestra en el cuadro 2 .

Todas las variables no deterministas presentan los signos esperados y son significativas al $5 \%$ o el $1 \%$, e incluso conjuntamente (estadístico F). Las pruebas de diagnóstico practicadas sobre: la especificación del modelo (prueba RESET Ramsey), la presencia de cambio estructural (Chow) y la existencia de multicolinealidad (factor de inflación de la varianza), junto con los criterios tradicionales de selección (criterio de información de Akaike y criterio de información

CUADRO 1

Pruebas de raíz unitaria y estacionarias

\begin{tabular}{|c|c|c|c|c|c|c|c|c|}
\hline \multirow{2}{*}{ Variable } & \multicolumn{4}{|c|}{$\mathrm{ADF}$} & \multicolumn{4}{|c|}{ PP } \\
\hline & Retraso & Términos establecidos & Prueba & Valor crítico $10 \%$ & Retraso & Términos establecidos & Prueba & Valor crítico $10 \%$ \\
\hline LY & 1 & $\mathrm{~N}$ & 0,70 & $-1,61$ & 2 & CT & $-3,15$ & $-3,17$ \\
\hline $\mathrm{D}(\mathrm{LY})$ & 0 & $\mathrm{~N}$ & $-10,78$ & $-1,61$ & 11 & $\mathrm{~N}$ & $-11,76$ & $-1,61$ \\
\hline LX & 2 & $\mathrm{~N}$ & 1,64 & $-1,61$ & 18 & $\mathrm{~N}$ & 1,56 & $-1,61$ \\
\hline $\mathrm{D}(\mathrm{LX})$ & 1 & $\mathrm{~N}$ & $-9,52$ & $-1,61$ & 21 & $\mathrm{~N}$ & $-8,45$ & $-1,61$ \\
\hline LI & 0 & $\mathrm{~N}$ & 0,59 & $-1,61$ & 57 & $\mathrm{~N}$ & 1,25 & $-1,61$ \\
\hline $\mathrm{D}(\mathrm{LI})$ & 0 & $\mathrm{~N}$ & $-7,77$ & $-1,61$ & 57 & $\mathrm{~N}$ & $-12,88$ & $-1,61$ \\
\hline LG & 4 & $\mathrm{~N}$ & 0,82 & $-1,61$ & 15 & $\mathrm{~N}$ & 1,41 & $-1,61$ \\
\hline $\mathrm{D}(\mathrm{LG})$ & 3 & $\mathrm{~N}$ & $-3,21$ & $-1,61$ & 28 & $\mathrm{~N}$ & $-15,62$ & $-1,61$ \\
\hline LM2 & 0 & $\mathrm{CT}$ & $-2,31$ & $-3,17$ & 0 & $\mathrm{CT}$ & $-2,31$ & $-3,17$ \\
\hline $\mathrm{D}(\mathrm{LM} 2)$ & 1 & $\mathrm{~N}$ & $-3,02$ & $-1,61$ & 2 & $\mathrm{~N}$ & $-6,42$ & $-1,61$ \\
\hline \multirow{2}{*}{ Variable } & \multicolumn{4}{|c|}{ DF-GLS } & \multicolumn{4}{|c|}{ KPSS } \\
\hline & Retraso & Términos establecidos & Prueba & Valor crítico $10 \%$ & Retraso & Términos establecidos & Prueba & Valor crítico \\
\hline LY & 1 & $\mathrm{C}$ & $-0,59$ & $-1,61$ & 5 & $\mathrm{CT}$ & 0,20 & 0,12 \\
\hline $\mathrm{D}(\mathrm{LY})$ & 0 & $\mathrm{CT}$ & $-7,74$ & $-2,87$ & 48 & $\mathrm{C}$ & 0,39 & 0,35 \\
\hline LX & 2 & $\mathrm{CT}$ & $-1,46$ & $-2,87$ & 5 & $\mathrm{CT}$ & 0,23 & 0,12 \\
\hline $\mathrm{D}(\mathrm{LX})$ & 1 & $\mathrm{CT}$ & $-8,60$ & $-2,87$ & 18 & $\mathrm{C}$ & 0,15 & 0,35 \\
\hline LI & 0 & $\mathrm{CT}$ & $-2,44$ & $-2,86$ & 5 & $\mathrm{CT}$ & 0,22 & 0,12 \\
\hline $\mathrm{D}(\mathrm{LI})$ & 0 & $\mathrm{CT}$ & $-5,62$ & $-2,87$ & 58 & $\mathrm{CT}$ & 0,50 & 0,12 \\
\hline LG & 4 & $\mathrm{CT}$ & $-1,78$ & $-2,88$ & 5 & $\mathrm{CT}$ & 0,21 & 0,12 \\
\hline $\mathrm{D}(\mathrm{LG})$ & 3 & $\mathrm{CT}$ & $-2,21$ & $-2,88$ & 15 & $\mathrm{C}$ & 0,14 & 0,35 \\
\hline LM2 & 0 & $\mathrm{CT}$ & $-1,14$ & $-2,86$ & 6 & $\mathrm{CT}$ & 0,23 & 0,12 \\
\hline $\mathrm{D}$ (LM2) & 1 & $\mathrm{C}$ & $-2,61$ & $-1,61$ & 3 & $\mathrm{C}$ & 0,42 & 0,35 \\
\hline
\end{tabular}

Fuente: elaboración propia sobre la base de datos del Instituto Brasileño de Geografía y Estadística/Sistema de Cuentas Nacionales (IBGE/sCN) y del Boletín del Banco Central del Brasil.

Notas: $\mathrm{N}=$ ninguno; $\mathrm{C}=$ constante; y $\mathrm{CT}$ = constante y tendencia lineal. En las pruebas de Dickey-Fuller aumentada (ADF) y Dickey-Fuller ajustada por tendencia (DF-GLS), la cantidad inicial de rezagos para cada serie se definió según el criterio de información de Schwarz. Para las pruebas de Phillips-Perron (PP) y la prueba estacionaria de KPSs (propuesta por Kwiatkowski, Phillips, Schmidt y Shin) se aplicó el criterio de Newey-West. Una $\mathrm{L}$ antes del nombre de cada variable indica su forma logarítmica.

Las letras DL denotan la primera diferencia de los logaritmos. 
CUADRO 2

\section{Modelo en primeras diferencias}

Variable dependiente: DLY

Método: mínimos cuadrados ordinarios (MCO)

Observaciones: 59

\begin{tabular}{|c|c|c|c|c|c|}
\hline Variable & $\mathrm{C}$ & DLX & DLI & DLG & DLM2 \\
\hline Coeficiente & $-0,0054$ & 0,1753 & 0,3228 & 0,2087 & 0,3312 \\
\hline Error estándar & 0,0076 & 0,0579 & 0,1151 & 0,0556 & 0,1537 \\
\hline Estadístico T & $-0,7094$ & 3,0296 & 2,8038 & 3,7564 & 2,1547 \\
\hline Factor de inflación de la varianza & & 1,0270 & 1,0060 & 1,1810 & 1,1690 \\
\hline $\mathrm{R}^{2}$ & 0,4658 & \multicolumn{3}{|c|}{ Durbin-Watson } & 2,3652 \\
\hline $\mathrm{R}^{2}$ ajustado & 0,4262 & \multirow{2}{*}{\multicolumn{2}{|c|}{ Breusch-Godfrey }} & Rezagos: 2 & 3,2573 \\
\hline Error estándar (ecuación) & 0,0547 & & & Rezagos: 4 & 3,2877 \\
\hline Probabilidad logarítmica & 90,3107 & \multirow{3}{*}{\multicolumn{2}{|c|}{ Prueba de ARCH }} & Rezagos: 1 & 2,3725 \\
\hline Criterio de información de Akaike & $-2,9618$ & & & Rezagos: 2 & 3,4820 \\
\hline Criterio de información de Schwarz & $-2,7158$ & & & Rezagos: 4 & 4,0796 \\
\hline Estadístico F & 11,7699 & \multicolumn{3}{|c|}{ Prueba de White } & 15,4668 \\
\hline Prueba de Chow & 3,0986 & \multicolumn{3}{|c|}{ Prueba de Breusch-Pagan } & 4,3934 \\
\hline Prueba RESET de Ramsey & 1,8084 & \multicolumn{3}{|c|}{ Prueba de Doornik-Hansen } & 4,7683 \\
\hline
\end{tabular}

Fuente: elaboración propia sobre la base de datos del Instituto Brasileño de Geografía y Estadística/Sistema de Cuentas Nacionales (IBGE/SCN) y del Boletín del Banco Central del Brasil.

Pruebas Durbin-Watson y Breusch-Godfrey para detectar problemas de autocorrelación.

Pruebas de White y Breusch-Pagan para detectar problemas de heterocedasticidad.

Prueba de diagnóstico sobre la especificación del modelo Prueba de RESET de Ramsey.

Prueba de ARCH para detectar problemas de heterocedasticidad condicional autorregresiva.

Prueba de Doornik-Hansen para detectar problemas de autocorrelación y normalidad.

Prueba de Chow de diagnóstico sobre la presencia de cambio estructural.

Las letras DL denotan la primera diferencia de los logaritmos.

C: constante.

de Schwarz), validan la parametrización elegida. Las pruebas practicadas en los residuales para detectar problemas de autocorrelación (Durbin-Watson y Breusch-Godfrey), heterocedasticidad (White, BreuschPagan), heterocedasticidad condicional autorregresiva (ARCH) y normalidad (Doornik-Hansen) no mostraron señales de los respectivos problemas. Las variables que figuran a la derecha de la ecuación de regresión explican aproximadamente el $47 \%$ de la variación del PIB, siendo la oferta monetaria responsable de la mayor repercusión: un incremento de un $1 \%$ en la oferta monetaria eleva el PIB en un $0,33 \%$.

$\mathrm{El}$ análisis de la dinámica a corto plazo presentado en el cuadro 2 - tras haber eliminado las tendencias en las variables por diferenciación- aportó información relevante sobre el comportamiento a largo plazo. Como todas las variables en cuestión son I(1), es posible la cointegración. Por consiguiente, se integraron las dinámicas a corto plazo y los equilibrios a corto plazo a partir de la teoría desarrollada en Granger (1981) y en Engle y Granger (1987).

La regresión de las variables estáticas expresadas como niveles (véase el cuadro 3 ) forma parte del procedimiento de dos etapas desarrollado por Engle-Granger
(EG) para probar la cointegración. Si las variables están cointegradas (el elemento residual es estacionario o I(0)), entonces es posible obtener parámetros constantes a largo plazo y el término de corrección de error para la regresión a corto plazo.

Aunque las pruebas de diagnóstico que se practicaron validan el modelo a largo plazo, los residuales pueden presentar autocorrelación, por lo que los errores estándar (y estadísticos T) que se observan se corrigieron por medio de la matriz de covarianzas consistentes y la prueba de heterocedasticidad y autocorrelación de White (HAC). Las variables LX y LI, significativas al 5\%, serán significativas al $1 \%$, mientras que LG es significativa al $1 \%$ y LM2 no es significativa en cualquiera de los dos casos (con o sin corrección) ${ }^{10}$.

Para superar el problema de la autocorrelación residual, se estimó una regresión dinámica a largo plazo para un modelo autorregresivo con rezagos distribuidos (ADL, por sus siglas en inglés) ${ }^{11}$. Este modelo

\footnotetext{
${ }^{10}$ En este caso la evidencia de autocorrelación es menos marcada.

${ }^{11}$ En el modelo estático con las transformaciones de Cochrane-Orcutt y Prais-Winsten, la significación de los parámetros se mantiene inalterada.
} 
CUADRO 3

\section{Modelo a largo plazo (EG)a}

Variable dependiente: LY

Método: mínimos cuadrados ordinarios (MCO)

Observaciones: 59 (ADL) y 60 Regresión estática

\begin{tabular}{|c|c|c|c|c|c|}
\hline Variable & $\mathrm{C}$ & $\mathrm{LX}$ & LI & LG & LM2 \\
\hline \multicolumn{6}{|c|}{ Regresión estática } \\
\hline Error estándar & 0,5207 & 0,0278 & 0,1193 & 0,0680 & 0,0543 \\
\hline Estadístico T & 1,2674 & 2,4735 & 2,6598 & 6,0805 & 0,9818 \\
\hline Error estándar (HAC) & 0,4864 & 0,0239 & 0,0847 & 0,1067 & 0,0634 \\
\hline Factor de inflación de la varianza & & 1,7170 & 2,3850 & 3,4210 & 4,6910 \\
\hline \multicolumn{6}{|c|}{ Autorregresivo con rezagos distribuidos } \\
\hline Coeficiente & 1,1726 & 0,0389 & 0,0793 & 0,5966 & 0,0316 \\
\hline Prueba de Wald (estadístico F) & 3,7628 & 1,5588 & 3,1683 & 10,9340 & 0,1173 \\
\hline $\mathrm{R}^{2}$ & 0,8743 & \multicolumn{2}{|c|}{ Durbin-Watson } & & 1,9849 \\
\hline Error estándar (ecuación) & 0,0468 & \multirow{2}{*}{\multicolumn{2}{|c|}{ Prueba de ARCH }} & Rezagos: 4 & 1,9216 \\
\hline Probabilidad logarítmica & 102,3642 & & & Rezagos: 1 & 0,0837 \\
\hline Criterio de Akaike & $-3,1310$ & & & Rezagos: 2 & 0,4808 \\
\hline Criterio de Schwarz & $-2,7789$ & & & Rezagos: 4 & 0,7408 \\
\hline Estadístico F & 37,8855 & \multicolumn{2}{|c|}{ Prueba de White } & & 57,9920 \\
\hline Prueba de Chow & 0,2095 & \multicolumn{2}{|c|}{ Prueba de Breusch-Pagan } & & 11,1197 \\
\hline Prueba RESET de Ramsey & 1,9947 & \multicolumn{2}{|c|}{ Prueba de Doornik-Hansen } & & 14,1386 \\
\hline
\end{tabular}

Fuente: elaboración propia sobre la base de datos del Instituto Brasileño de Geografía y Estadística/Sistema de Cuentas Nacionales (IBGE/SCN) y del Boletín del Banco Central del Brasil.

Notas: las estadísticas de diagnóstico se refieren al modelo autorregresivo con rezagos distribuidos (ADL) con rezago 1.

Pruebas Durbin-Watson y Breusch-Godfrey para detectar problemas de autocorrelación.

Pruebas de White y Breusch-Pagan para detectar problemas de heterocedasticidad.

Prueba de diagnóstico sobre la especificación del modelo Prueba de RESET de Ramsey.

Prueba de ARCH para detectar problemas de heterocedasticidad condicional autorregresiva.

Prueba de Doornik-Hansen para detectar problemas de autocorrelación y normalidad.

Prueba de Chow de diagnóstico sobre la presencia de cambio estructural.

ADL: modelo autorregresivo con rezagos distribuidos.

HAC: prueba de heterocedasticidad y autocorrelación de White.

$\mathrm{C}$ : constante.

a (EG): procedimiento de dos etapas desarrollado por Engle-Granger para probar la cointegración.

supera las pruebas de diagnóstico. En términos de significación de los parámetros, LX, LI y LG siguen siendo significativos (por lo menos al 10\%), aunque LM2 no lo es.

Con el fin de asegurar que las relaciones estimadas no sean espurias es necesario cointegrar las variables en estudio. El siguiente paso del procedimiento de Engle-Granger consiste en verificar si los residuales en la relación a largo plazo son estacionarios, para cuyo efecto se utiliza la prueba de Dickey-Fuller aumentada (ADF). No obstante, para contrastar esta hipótesis no es aconsejable utilizar los valores de los cuadros tradicionales. Como estos no se confeccionan para los valores estimados, aquí se utiliza el cuadro ajustado por los valores estimados y por el tamaño de la muestra según lo propone MacKinnon (1990). El valor de la prueba estadística $(-4,68)$ rechaza la presencia de una raíz unitaria con un nivel de confianza del $99 \%$, lo que indica la existencia de una relación a largo plazo entre variables.

La existencia de variables cointegradas significa que se puede utilizar el modelo de corrección de errores. Ello conecta los aspectos de las dinámicas a corto y largo plazo; en otras palabras, permite combinar las ventajas del "modelamiento" con variables expresadas como diferencias y como niveles.

Como se puede apreciar en el cuadro 4, las variables en estudio son diferenciadas y, por lo tanto, estacionarias (originalmente tenían una raíz unitaria). Para que la ecuación esté en equilibrio en el sentido de 
Variable dependiente: DLY

Método: mínimos cuadrados ordinarios (MCO)

Observaciones: 59

\begin{tabular}{lccccrr}
\hline Variable & C & DLX & DLI & DLG & DLM2 & TCE(-1) \\
\hline Coeficiente & $-0,0030$ & 0,1600 & 0,3438 & 0,2727 & 0,1965 & $-0,5972$ \\
Error estándar & 0,0062 & 0,0587 & 0,0996 & 0,0305 & 0,0960 & 0,1044 \\
Estadístico T & $-0,4912$ & 2,7233 & 3,4532 & 8,9286 & 2,0465 & $-5,7199$ \\
Factor de inflación de la varianza & & 1,0310 & 1,0080 & 1,2780 & 1,2250 & 1,1140 \\
\hline $\mathrm{R}^{2}$ & 0,6248 & & Durbin-Watson & Rezagos: 2 & 1,9008 \\
$\mathrm{R}^{2}$ ajustado & 0,5894 & & Breusch-Godfrey & Rezagos: 4 & 1,8903 \\
Error estándar (ecuación) & 0,0463 & & & Rezagos: 1 & 0,1385 \\
Probabilidad logarítmica & 100,7344 & & Prueba de ARCH & Rezagos: 2 & 0,1474 \\
Criterio de Akaike & $-3,2113$ & & & Rezagos: 4 & 0,1624 \\
Criterio de Schwarz & $-3,0001$ & & & & 10,1296 \\
Estadístico F & 17,6509 & & Prueba de White & Prueba de Breusch-Pagan & & 2,8016 \\
Prueba de Chow & 0,7125 & & Prueba de Doornik-Hansen & & 17,6178 \\
Prueba RESET de Ramsey & 2,1170 & & & &
\end{tabular}

Fuente: elaboración propia sobre la base de datos del Instituto Brasileño de Geografía y Estadística/Sistema de Cuentas Nacionales (IBGE/SCN) y del Boletín del Banco Central del Brasil.

Notas:

Pruebas Durbin-Watson y Breusch-Godfrey para detectar problemas de autocorrelación.

Pruebas de White y Breusch-Pagan para detectar problemas de heterocedasticidad.

Prueba de diagnóstico sobre la especificación del modelo Prueba de RESET de Ramsey.

Prueba de ARCH para detectar problemas de heterocedasticidad condicional autorregresiva.

Prueba de Doornik-Hansen para detectar problemas de autocorrelación y normalidad.

Prueba de Chow de diagnóstico sobre la presencia de cambio estructural

Las letras DL denotan la primera diferencia de los logaritmos.

C: constante.

a (EG): procedimiento de dos etapas desarrollado por Engle-Granger para probar la cointegración.

estar en el mismo nivel de integración, es necesario que el término de corrección de error sea $\mathrm{I}(0)$. Así, la cointegración entre las variables - en el sentido propuesto en Engle y Granger (1987) - exige la existencia de un modelo de corrección de errores, y viceversa. Todas las elasticidades son significativas (por lo menos al 5\%) y cercanas a los valores obtenidos mediante el modelo habitual expresado en forma de primera diferencia. No obstante, el coeficiente del término de corrección de error - que mide la distancia entre $\mathrm{X}$ e $\mathrm{Y}$ en relación con el equilibrio a largo plazo y expone así la velocidad de ajuste de las variables a desequilibrios ocasionales-es de aproximadamente el $60 \% 12$.

Para un análisis más sólido, se utilizó el enfoque descrito en Johansen (1988 y 1991) y en Johansen y Juselius (1990) con el fin de verificar la existencia de cointegración y las relaciones existentes para el equilibrio a largo

\footnotetext{
${ }^{12}$ Se espera que el signo negativo asegure que cualquier desequilibrio que se produzca quede compensado por un movimiento opuesto en la variable objeto de explicación.
}

plazo. El procedimiento de Johansen es un método más general de máxima verosimilitud que utiliza un sistema de ecuaciones dinámicas, específicamente, un modelo de autorregresión vectorial. Con el enfoque sistémico propuesto por Johansen se logra identificar no solo la presencia de cointegración, sino también, de confirmarse, la cantidad de vectores cointegradores y su especificación.

La cantidad de rezagos se determinó según los criterios de la máxima verosimilitud modificada, el error final de predicción, el criterio de información de Akaike, el criterio de información de Schwarz y el criterio de información de Hannan-Quinn, mientras que la decisión de incluir términos deterministas se basó en un análisis visual de la serie y en el criterio de Pantula. Para verificar si existe cointegración y, a la vez, si existe la cantidad de vectores, se utilizan las pruebas de la traza y de la raíz característica máxima (véase el cuadro 5).

Como ambas pruebas indican la existencia de un vector de cointegración, es posible estimar el vector de corrección de errores (VEC). En la ecuación (2) se muestran las elasticidades a largo plazo obtenidas del vector de cointegración: 


$$
\begin{aligned}
& L Y=1,1972+\underset{(0,0420)}{01099} L X+\underset{c}{0,7067 L I} \\
& +0,4052 L G+0,0322 L M 2 \\
& \begin{array}{ll}
(0,1490) & (0,1045) \\
{[-2,7197]} & {[-0,3077]}
\end{array}
\end{aligned}
$$

Muestra: marzo de 1992 - abril de 2005; rezagos: 1 a 5; los datos presentan una tendencia determinista.

Las estimaciones de los parámetros en la ecuación (2) son los coeficientes de cointegración normalizados, los valores entre paréntesis son errores estándar, y los estadísticos $\mathrm{T}$ constan entre corchetes. Todas las elasticidades presentan los signos esperados en la teoría y son estadísticamente significativas (al 5\%), con excepción, una vez más, de LM2.

En el cuadro 6 se resumen los resultados obtenidos del VEC, en particular el término de corrección de error de cada ecuación y los diagnósticos básicos del modelo en su conjunto. Las cifras indican los coeficientes de ajuste del equilibrio a largo plazo obtenidos de cada una de las cinco ecuaciones múltiples del VEC. La significación del término de corrección de errores de cada ecuación indica que la variable dependiente se ajusta en respuesta a un desequilibrio entre dicha variable y las

\begin{tabular}{|c|c|c|c|}
\hline \multicolumn{4}{|c|}{ Prueba de la traza } \\
\hline $\begin{array}{l}\text { Hipótesis } \\
\text { Número de corrección de errores }\end{array}$ & Valor propio & $\begin{array}{l}\text { Estadístico } \\
\text { de traza }\end{array}$ & $\begin{array}{c}0,05 \\
\text { Valor crítico }\end{array}$ \\
\hline Ninguno & 0,4969 & 76,9029 & 69,8189 \\
\hline Como máximo 1 & 0,3780 & 39,8108 & 47,8561 \\
\hline Como máximo 2 & 0,1523 & 14,1673 & 29,7971 \\
\hline Como máximo 3 & 0,0903 & 5,2455 & 15,4947 \\
\hline Como máximo 4 & 0,0025 & 0,1336 & 3,8415 \\
\hline \multicolumn{4}{|c|}{ Prueba de la raíz característica máxima } \\
\hline $\begin{array}{l}\text { Hipótesis } \\
\text { Número de corrección de errores }\end{array}$ & Raíz característica & $\begin{array}{l}\text { Estadístico de la raíz } \\
\text { característica máxima }\end{array}$ & $\begin{array}{c}0,05 \\
\text { Valor crítico }\end{array}$ \\
\hline Ninguno & 0,4969 & 37,0921 & 33,8769 \\
\hline Como máximo 1 & 0,3780 & 25,6435 & 27,5843 \\
\hline Como máximo 2 & 0,1523 & 8,9218 & 21,1316 \\
\hline Como máximo 3 & 0,0903 & 5,1119 & 14,2646 \\
\hline Como máximo 4 & 0,0025 & 0,1336 & 3,8415 \\
\hline
\end{tabular}

CUADRO 5

Pruebas de cointegración

Fuente: elaboración propia sobre la base de datos del Instituto Brasileño de Geografía y Estadística/Sistema de Cuentas Nacionales (I BGE/sCN) y del Boletín del Banco Central del Brasil.

CUADRO 6

Resumen de resultados del VECa

\begin{tabular}{lccccc}
\hline & $\mathrm{D}(\mathrm{LY})$ & $\mathrm{D}(\mathrm{LX})$ & $\mathrm{D}(\mathrm{LI})$ & $\mathrm{D}(\mathrm{LG})$ & $\mathrm{D}(\mathrm{LM})$ \\
\hline Término de corrección de errores & $-0,7540$ & 0,9970 & 0,4946 & $-0,7177$ & $-0,2112$ \\
Error estándar & $(0,2490)$ & $(0,5846)$ & $(0,2423)$ & $(0,3805)$ & $(0,2156)$ \\
Estadístico T & {$[-3,0285]$} & {$[1,7053]$} & {$[2,0379]$} & {$[-1,8858]$} & {$[-0,9794]$} \\
$\mathrm{R}^{2}$ ajustado & 0,6757 & 0,4361 & 0,4121 & 0,8157 & 0,4696 \\
Error estándar (ecuación) & 0,0405 & 0,0952 & 0,0411 & & 0,0620 \\
\hline Estadístico LM (autocorrelación) & 24,2728 & & & \\
White (heterocedasticidad) & 801,1020 & & & & \\
Prueba de Lutkepohl (normal) & 2,8940 & & & & \\
\hline
\end{tabular}

Fuente: elaboración propia sobre la base de datos del Instituto Brasileño de Geografía y Estadística/Sistema de Cuentas Nacionales (IBGE/sCN) y del Boletín del Banco Central del Brasil.

a Vector de corrección de errores.

Las letras denotan la primera diferencia de los logaritmos. 
variables independientes, lo que entonces es indicativo de la endogeneidad.

Los resultados estadísticos de la prueba del VEC rechazan la presencia de autocorrelación, heterocedasticidad y no normalidad en los residuales. En la matriz de ajustes, solamente los términos de corrección de error de las ecuaciones de producto e inversión son significativos (hasta el 5\%), lo que evidencia la (débil) exogeneidad de las exportaciones y los gastos públicos en el modelo. Si bien la oferta monetaria no se ajusta a los desequilibrios a largo plazo, no es significativa en la ecuación a largo plazo.

El análisis de los desequilibrios a corto plazo y su interacción con la dinámica a largo plazo, realizado en función de la metodología de Engle-Granger y el procedimiento de Johansen, aporta algunas conclusiones interesantes. En el período examinado se observan empíricamente para la economía brasileña los signos indicados por la teoría; las variables explicativas —es decir, exportaciones, inversión y gastos del gobiernoson todas significativas tanto en el corto como en el largo plazo. La oferta monetaria solo es significativa en la dinámica a corto plazo, por lo que no parece probable que la política monetaria afecte en forma sostenida al crecimiento económico del Brasil. Ello obedece a que los cambios monetarios, definidos en términos amplios, no tienen influencia estadística en el comportamiento del PIB real en el largo plazo.

Según los coeficientes estimados de la ecuación de regresión (2), por cada aumento de un $1 \%$ en el gasto público real, el PIB real crece un $0,40 \%$. Así pues, bajo el entendido de que la recaudación impositiva en las tres esferas del gobierno representa aproximadamente el $40 \%$ del PIB, un incremento en el consumo público corriente del orden de un $1 \%$ generaría un crecimiento de aproximadamente un $0,16 \%$ en la recaudación impositiva, lo que empeoraría el déficit del sector público. Dada la pesada carga tributaria que actualmente soporta la economía brasileña (aproximadamente del $40 \%$ ) y la elevada relación entre la deuda pública y el PIB (también cercana al 40\%), en las condiciones actuales el gobierno no puede estimular permanentemente el crecimiento económico mediante el aumento de su consumo corriente. Las exportaciones representan la única fuente "autónoma" de demanda que podría inducir la aceleración de la expansión económica. En otras palabras, la economía brasileña debe adoptar un modelo de crecimiento impulsado por las exportaciones.

Salvo en lo que se refiere a la oferta monetaria, los resultados expuestos en este trabajo son muy similares a los obtenidos por Atesoglu (2002). Las relaciones de causalidad respaldan el enfoque keynesiano que se analizó en la sección II, en el que las exportaciones y el consumo público son las fuentes fundamentales del crecimiento económico a largo plazo. No obstante, dada la grave crisis fiscal imperante en el Brasil, impulsar el dinamismo económico mediante una política destinada a ampliar el consumo público corriente no parece posible. Para que en la economía brasileña se reanude una rápida expansión económica es necesario adoptar un modelo de crecimiento impulsado por las exportaciones.

\section{2. ¿Es endógena la tasa natural de crecimiento de la economía brasileña?}

En la subsección 1 se demostró que la tasa de crecimiento observada en la economía brasileña está determinada por el aumento de la demanda agregada. En esta subsección se avanza aún más con este razonamiento al demostrar que la tasa natural de crecimiento ${ }^{13}$ también se ajusta a la tasa real de expansión de la economía en el largo plazo. Esto significa que el incremento de la demanda agregada determina no solo el dinamismo de la tasa real de avance de la economía brasileña, sino también la dinámica de la tasa natural de crecimiento que convencionalmente se vincula al progreso tecnológico y al acrecentamiento de la fuerza de trabajo.

Esta subsección se basa en un estudio realizado por Ledesma y Thirlwall (2002). Al utilizar el concepto definido por Okun (1962, citado por Ledesma y Thirlwall, 2002), la tasa natural de crecimiento $\left(g_{n}\right)$ es lo que mantiene constante el nivel de desempleo. Okun (1962, citado por Ledesma y Thirlwall, 2002) utiliza la siguiente especificación para el cambio en la tasa de desempleo porcentual:

$$
\Delta \% U=a-b(g)
$$

\footnotetext{
${ }^{13}$ En los antiguos modelos neoclásicos de crecimiento, representados por Solow (1956), la tasa natural de crecimiento era exógena y estaba determinada por factores de la oferta, entre ellos la tasa de progreso tecnológico y el incremento de la fuerza de trabajo. En la "nueva teoría del crecimiento", que tiene sus orígenes en los influyentes trabajos de Romer (1986) y Lucas (1988), la tasa natural de crecimiento se convierte en una variable endógena en el sentido de que la tasa de progreso tecnológico está determinada por el modelo mismo. No obstante, no es este el significado con el que se utiliza el término "endógeno" en este artículo. Aquí, la expresión "tasa natural de crecimiento endógeno" significa una tasa de expansión de la producción real determinada por la tasa de incremento de la demanda agregada en el largo plazo. Véase Libanio (2009), donde consta una interpretación similar del término "endógeno".
} 
donde $U$ es el nivel de desempleo, $g$ es la tasa de crecimiento de la producción y $a$ y $b$ son dos constantes. En la ecuación (3), cuando $\Delta \% U=0$, la tasa natural de crecimiento está definida por $a / b$.

En épocas de crecimiento económico lento algunas personas no buscan empleo, por ello es posible que se subestime el coeficiente $a$. En este caso, también se estaría subvalorando la tasa natural de expansión económica. Asimismo, en períodos de dinamismo económico parte del empleo adicional necesario para aumentar la producción proviene de la mano de obra previamente no aprovechada, y también de las horas extra. Así, se estima a $b$ por debajo de su valor, lo que conduce a la sobrevaloración de la tasa natural de crecimiento. En consecuencia, dicha tasa puede quedar subvalorada o sobrevalorada según cuál de esos dos efectos predomine.

En un intento por eludir estos problemas, Thirlwall (1969) desarrolló un enfoque distinto para estimar la tasa natural de crecimiento:

$$
g=a_{1}-b_{1}(\Delta \% U)
$$

En la ecuación (4), cuando la variación de la tasa de desempleo es cero (0), tenemos que:

$$
g=a_{1}
$$

Así, la tasa natural de crecimiento está definida por la ordenada en el origen de la ecuación de regresión. El problema de utilizar la ecuación (4) radica en que la tasa natural de crecimiento es endógena, por lo que los coeficientes estimados están sesgados.

Una vez estimada dicha tasa natural, se puede crear una variable ficticia que toma el valor 1 cuando el crecimiento económico real supera la tasa natural estimada mediante las ecuaciones (3) o (4), y el valor 0 en el caso contrario. Con la incorporación de variables ficticias se llega a la siguiente ecuación de regresión:

$$
g=a_{2}+b_{2} D+c_{2}(\Delta \% U)
$$

donde $D$ es la variable ficticia. Al especificar la ecuación (6), se estiman dos tasas naturales de crecimiento. La primera se estima para aquellos períodos en que la tasa de crecimiento se sitúa sobre la tasa natural obtenida mediante la ecuación (4). En este caso, la tasa natural de crecimiento equivale a $a_{2}+b_{2}$. La segunda se estima para los períodos en que la tasa de crecimiento observada es inferior a la tasa natural obtenida mediante la ecuación (4). En este caso, la tasa natural es $a_{2}$.
Cabe esperar que la "tasa natural" no varíe cuando se modifica la tasa real de crecimiento. De ser así, el coeficiente de la variable ficticia no debería ser estadísticamente distinto de 0 . De lo contrario, la tasa natural $\left(g_{n}\right)$ es endógena y responde a cambios que se pueden producir en la tasa real de crecimiento $(g)$.

En este estudio, la base de datos utilizada para llevar adelante el análisis de regresión contiene variables de PIB y desempleo. El nivel de desempleo se tomó de la Encuesta Mensual de Empleo del IBGE, y las cifras mensuales originales se transformaron en datos trimestrales mediante el cálculo de la media aritmética de los tres meses de cada trimestre ${ }^{14}$. El índice en cadena del PIB se basa en el Sistema de Cuentas Nacionales del IBGE (IBGE/SCN $)^{15}$. El período de análisis se extiende desde el primer trimestre de 1980 hasta el último de $2002^{16}$. Como ambas variables se transformaron en tasas de crecimiento, se perdió la primera observación de cada serie, lo que deja 91 observaciones para el análisis empírico.

Mediante la utilización de las series de datos trimestrales, en el cuadro 7 se presentan las estimaciones de la tasa natural de crecimiento obtenidas de las ecuaciones (3) y (4):

Las tasas de crecimiento generadas por cada una de las ecuaciones son muy similares, lo que indica que la tasa natural estimada es robusta a pesar de los posibles problemas mencionados anteriormente.

Una tasa natural de crecimiento de alrededor de un $0,60 \%$ por trimestre importa una tasa anualizada cercana a un $2,50 \%$. Así, las ecuaciones de regresión utilizadas indican que la tasa de crecimiento que habría mantenido constante el desempleo en el Brasil entre los años 1980 y 2002 se aproximaba a un $2,50 \%$.

En el cuadro 8 se ilustran los resultados empíricos obtenidos de la ecuación de regresión (6). El símbolo MA significa que la tasa de crecimiento del PIB es una media móvil de tres trimestres.

\footnotetext{
${ }^{14}$ A partir de datos mensuales se dividió cada año en cuatro trimestres, para cuyo efecto se sumaron las cifras de desempleo correspondientes a los tres meses y se dividió por tres. Tasa de desempleo del primer trimestre (enero + febrero + marzo) / 3 ; tasa de desempleo del segundo trimestre (abril + mayo + junio) / 3; tasa de desempleo del tercer trimestre (julio + agosto + septiembre) / 3; tasa de desempleo del cuarto trimestre (octubre + noviembre + diciembre) $/ 3$.

${ }^{15}$ Serie en cadena del índice trimestral móvil, ajustada estacionalmente, con una media $1990=100$.

16 El período de análisis concluye en 2002, dado que en 2003 se introdujo un cambio metodológico en la base de datos de la Encuesta Mensual de Empleo, lo que hizo imposible extender las pruebas econométricas al período más reciente.
} 


\begin{tabular}{|c|c|c|c|c|c|c|}
\hline & Método & $\begin{array}{l}\text { Ordenada } \\
\text { en el origen }\end{array}$ & Pendiente & DW & $\mathrm{R}^{2}$ ajustado & NRG \\
\hline Ecuación (3) & $\mathrm{RR}$ & $\begin{array}{c}1,61 \\
(0,99)\end{array}$ & $\begin{array}{l}-2,70^{* * *} \\
(3,49)\end{array}$ & 2,32 & 0,11 & 0,60 \\
\hline Ecuación (4) & MCO & $\begin{array}{l}0,59 * * * \\
(2,99)\end{array}$ & $\begin{array}{l}-0,053^{* * * *} \\
(4,12)\end{array}$ & 1,89 & 0,15 & 0,59 \\
\hline
\end{tabular}

Fuente: elaboración propia sobre la base de datos del Instituto Brasileño de Geografía y Estadística/Sistema de Cuentas Nacionales (IBGE/SCN). Notas: *** Significativo al $1 \%$. RR: método de regresión robusta utilizado para corregir problemas de no normalidad de residuales y heterocedasticidad. MCO: mínimos cuadrados ordinarios; DW: valor de la prueba de Durbin-Watson para la autocorrelación de primer orden; $\mathrm{R}^{2}$ aj.: $\mathrm{R}^{2}$ ajustado, y NRG: tasa natural de crecimiento.

CUADRO 8

Estimación de la tasa natural de crecimiento mediante
las ecuaciones de Okun y Thirlwall con una variable ficticia

\begin{tabular}{lcccccccc}
\hline & Método & $\begin{array}{c}\text { Ordenada } \\
\text { en el origen }\end{array}$ & $\begin{array}{c}\text { Coeficiente } \\
\text { ficticio }\end{array}$ & Pendiente & DW & $\mathrm{R}^{2}$ ajustado & NRG $\left(g<g_{n}\right)$ & NRG $\left(g>g_{n}\right)$ \\
\hline Ecuación (6) & MCO & $\begin{array}{c}-0,84 * * * \\
(-4,40)\end{array}$ & $\begin{array}{c}2,85^{* * *} \\
(10,40)\end{array}$ & $\begin{array}{c}0,03^{* * *} \\
(-3,35)\end{array}$ & 2,28 & 0,61 & $-0,84$ & 2,01 \\
Ecuación (6) MA & PWER & $\begin{array}{c}-0,26^{*} \\
(-1,66)\end{array}$ & $\begin{array}{c}1,56^{* * *} \\
(10,26)\end{array}$ & $\begin{array}{c}0,011^{* *} \\
(-2,14)\end{array}$ & 1,82 & 0,54 & $-0,26$ & 1,3 \\
\hline
\end{tabular}

Fuente: elaboración propia sobre la base de datos del Instituto Brasileño de Geografía y Estadística/Sistema de Cuentas Nacionales (IBGE/SCN). Notas: *** Significativo al $1 \%$; ** significativo al $5 \%$; significativo al $10 \%$.

MCO: mínimos cuadrados ordinarios; PWER: método de Prais-Wisten con errores robustos para corregir problemas de autocorrelación y heterocedasticidad; DW: valor de la prueba de Durbin-Watson para la autocorrelación de primer orden; NRG: tasa natural de crecimiento, y MA: ecuación de regresión con medias móviles de tres trimestres.

Los resultados de la ecuación de regresión (6) indican que la tasa natural (de crecimiento) responde al crecimiento real de la economía. Las cifras expuestas en la primera fila del cuadro 8 permiten inferir que en períodos de crecimiento económico rápido, la tasa natural es de aproximadamente un $8 \%$, mientras que en períodos de crecimiento débil o recesión, la tasa natural es en realidad negativa, ubicándose en aproximadamente un $-3,5 \%$.

Cabe recordar que los datos son trimestrales, por lo que el rango de variación es amplio. El uso de medias móviles presenta, entre otras, la ventaja de que dichas medias hacen menos marcadas las fluctuaciones, tal como se puede apreciar a partir de los resultados expuestos en la segunda fila del cuadro 8 . En este caso, la tasa natural de crecimiento anual en "épocas buenas" sería de aproximadamente un $5,2 \%$, mientras que en "épocas malas" se ubicaría en un nivel cercano al $-1 \%$.

Las pruebas demuestran que la tasa natural de crecimiento de la economía brasileña es una variable endógena y, por consiguiente, puede verse afectada por las condiciones de la demanda imperantes en la economía. Las estimaciones correspondientes a la tasa natural de crecimiento en "épocas buenas" varían entre un 5,2\% y un $8 \%$ por año, lo que indica que la economía brasileña podría expandirse a tasas anuales claramente superiores a un 3,5\%, sin generar presiones de índole inflacionaria. Los resultados empíricos aportan evidencias de que el crecimiento económico del Brasil no está restringido por la oferta, sino por la demanda. 
IV

\section{Análisis empírico de la relación entre el tipo de cambio real (TCR) y la elasticidad- ingreso de las exportaciones}

En la sección III se demostró que las tasas observada y natural de crecimiento de la economía brasileña están determinadas por el incremento de la demanda agregada. También se señaló que este incremento es impulsado por el acrecentamiento de las exportaciones y de los gastos públicos (dado que la inversión es endógena). No obstante, debido a la crisis fiscal, en el Brasil no es factible un modelo de crecimiento promovido por la expansión fiscal, lo que significa que este solo puede ser impulsado por una expansión constante de las exportaciones.

¿Qué condiciones deben reunirse para que haya una expansión sólida y constante de las exportaciones brasileñas o en otras economías capitalistas? En el largo plazo, la tasa de crecimiento de las exportaciones de un país o una región es determinada por la elasticidad-ingreso mundial de las exportaciones, multiplicada por la tasa de crecimiento de los ingresos en el resto del mundo. La elasticidad-ingreso de las exportaciones capta la influencia que tienen los factores ajenos al precio en la competitividad externa del país, tales como el contenido tecnológico de los productos exportados, el grado de diferenciación de los productos de exportación en comparación con los productos competidores en el mercado internacional, el valor agregado de dichos productos, entre otros. Cuanto mayor sea la elasticidad-ingreso de las exportaciones, mayor será la tasa de aumento de las exportaciones del país dada una tasa determinada de crecimiento de los ingresos mundiales. Se trata del canal por el que los factores de la oferta pueden afectar, mas no determinar, la tasa de crecimiento de la producción real a largo plazo 17 .

\footnotetext{
${ }^{17}$ La inclusión de factores de oferta en el análisis efectuado en este artículo no menoscaba el papel de la demanda agregada en tanto causa principal del crecimiento económico. La tasa de incremento de la producción real a largo plazo está determinada por la tasa de crecimiento de la demanda autónoma, que recibe la influencia de factores derivados de la oferta, aunque no está determinada por estos. Por otra parte, la inclusión de factores de la oferta en el análisis posibilita la identificación de un canal preciso mediante el cual el tipo de cambio real puede influir en el crecimiento de las exportaciones en el largo plazo. La conclusión del análisis, expuesta en los párrafos
}

Los países que se ubican en la llamada "frontera tecnológica", en general, deberían presentar una elasticidad-ingreso de las exportaciones superior a la de los países en desarrollo. Ello obedece a que los países más próximos a la "frontera tecnológica" tienden a exportar productos con mayor valor agregado y más alto contenido tecnológico que los países más alejados. Esta "brecha tecnológica" constituiría así un importante factor de la elasticidad-ingreso de las exportaciones $\mathrm{y}$, por consiguiente, de la tasa de crecimiento de las exportaciones a largo plazo (Dosi, Pavitt y Soete, 1990, pág. 26).

Sin embargo, en el material publicado - tanto teórico como empírico - sobre los factores determinantes de la elasticidad-ingreso de las exportaciones se ha descuidado la función que cumple el tipo de cambio real, que es uno de sus determinantes. De hecho, los trabajos empíricos sobre las variables que afectan al desempeño de las exportaciones se han limitado a estimar las elasticidadesprecio de las exportaciones; pero estas estimaciones han presentado el signo opuesto al pronosticado por la teoría o bien no han sido significativas.

No se ha realizado intento alguno de evaluar la existencia de una relación entre la elasticidad-ingreso de las exportaciones y el tipo de cambio real. En el material publicado parece respaldarse la hipótesis de que el tipo de cambio real (TCR) solo puede influir en el crecimiento económico a largo plazo mediante los efectos que produce en la predisposición de los consumidores locales y extranjeros a gastar sus ingresos en bienes nacionales o importados. En la literatura se desatiende la posible repercusión del TCR en la estructura productiva de la economía y, por ende, en la elasticidad-ingreso de las exportaciones.

En el plano exclusivamente teórico, puede establecerse una relación entre el nivel del TCR y la

siguientes, es que para que se produzca una expansión sólida y continua de las exportaciones (que es condición necesaria para que el Brasil alcance altas tasas de crecimiento a largo plazo) es menester mantener subvalorado el tipo de cambio real. 
elasticidad-ingreso de las exportaciones mediante el modelo ricardiano de comercio internacional expuesto en Dornbusch, Fischer y Samuelson (1977). A partir de este modelo, el grado de especialización productiva de una economía - la cantidad de distintos tipos de bienes que produce la economía nacional- está determinado por la relación entre el salario real local y los salarios reales que se pagan en el mundo.

Cuanto más elevados sean los salarios reales que se pagan en la economía local en comparación con el resto del mundo, mayor será el grado de especialización productiva del país, o menor será la cantidad de distintos tipos de bienes que se producen en la economía local. Cuanto mayor sea el grado de especialización productiva, menor será la tasa de incremento de las exportaciones en función de una tasa dada de crecimiento de los ingresos mundiales (en otras palabras, menor será la elasticidadingreso de las exportaciones).

El TCR afecta al grado de especialización productiva de la economía, porque afecta directamente a los salarios reales. La valorización del TCR provoca, en general, un alza en los salarios, lo que aumenta los costos de producción del país en relación con los imperantes en el resto del mundo. Este proceso obliga a las actividades productivas que se realizan en la economía local a migrar al exterior, lo que conlleva la "desindustrialización" de la economía local, con repercusiones negativas para su capacidad exportadora.

Con el fin de evaluar si la elasticidad-ingreso de las exportaciones se ve afectada por el TCR y la brecha tecnológica ${ }^{18}$, se analizaron 30 países desarrollados y en desarrollo ${ }^{19}$ mediante una metodología de regresión que comprende dos pasos. En primer lugar, se estimaron los valores de las elasticidades-ingreso de las exportaciones de los países seleccionados en el período 1995-2005; luego se estimó la relación entre la elasticidad-ingreso de las exportaciones de un país y su TCR y brecha tecnológica.

La ecuación estimada en la primera etapa es la siguiente:

$$
X_{i}=c_{0}+c_{1} Q+c_{2} Y^{*}+\varepsilon_{i}
$$

donde $X_{i}$ es el valor real en dólares de las exportaciones del país $i^{\prime}, Y^{*}$ es el valor real en dólares del PIB del resto del mundo, $Q$ es un índice del TCR tomado como media del período 1995-2005 (1995 = 100), $c_{0}$ es una constante, $\varepsilon_{i}$ es el término de error, $c_{1}$ representa la elasticidadtipo de cambio de las exportaciones, y $c_{2}$ representa la elasticidad-ingreso de las exportaciones o, en otras palabras, cómo responden las exportaciones de cada país ante los cambios registrados en el PIB mundial ${ }^{20}$. Para todas las series se utilizaron datos trimestrales.

La estimación de la ecuación de la segunda etapa estuvo destinada a captar cualquier efecto generado por el TCR y la brecha tecnológica en la elasticidad-ingreso de las exportaciones. Con este propósito se estimó una regresión de los mínimos cuadrados ordinarios (MCO) de los valores de las elasticidades-ingreso de las exportaciones estimadas en la primera etapa en contraste con los índices de TCR y brecha tecnológica correspondientes a los países seleccionados en el período 1995-2005. La especificación del modelo permite la interacción entre el TCR y la brecha tecnológica al determinar la elasticidad-ingreso de las exportaciones. La incorporación de esta interacción hace posible analizar si el efecto de las variaciones registradas en el tipo de cambio en la elasticidad-ingreso de las exportaciones se ve afectado por dicha brecha. Cabría esperar que aquellos países que presentan una mayor brecha tecnológica respecto de los Estados Unidos de América compensen su desventaja en ese campo mediante la devaluación de la moneda. Para los países que se ubican más próximos a la frontera tecnológica se espera el resultado contrario: un mayor nivel de competitividad ajena al precio permite que dichos países mantengan una moneda valorizada y, por lo tanto, salarios reales más elevados.

Para el cálculo del índice del TCR se utilizaron datos trimestrales sobre el tipo de cambio nominal y los índices de precios al consumidor obtenidos de las Estadísticas financieras internacionales, y se los normalizó a 100 en 1995. A continuación, en el gráfico 1 se muestra la dispersión de la elasticidad-ingreso de las exportaciones y el índice del TCR.

En el gráfico 1 se ilustra la existencia de una relación no lineal entre la elasticidad-ingreso de las exportaciones

\footnotetext{
${ }^{20}$ De los 30 países examinados, 24 no presentaron problema alguno para la estimación de $c_{2}$ en lo que a nivel respecta. En los casos de Chile, Dinamarca, Noruega, Nueva Zelandia, Portugal y el Reino Unido de Gran Bretaña e Irlanda del Norte, las exportaciones y el PIB mundial no se cointegran, por lo que resulta imposible estimar el nivel correcto de la elasticidad-ingreso de las exportaciones. En otros dos países -Austria y México-, el índice del TCR a nivel es estacionario.
}

\footnotetext{
${ }^{18}$ El concepto de brecha tecnológica se debe a Fagerberg (1988).

19 Alemania, Argentina, Australia, Austria, Brasil, Canadá, Chile, Dinamarca, España, Estados Unidos de América, Federación de Rusia, Francia, Hungría, Indonesia, Italia, Malasia, México, Noruega, Nueva Zelandia, Países Bajos, Portugal, Reino Unido de Gran Bretaña e Irlanda del Norte, República Checa, República de Corea, Sudáfrica, Suecia, Suiza, Tailandia y Turquía.
} 
GRÁFICO 1

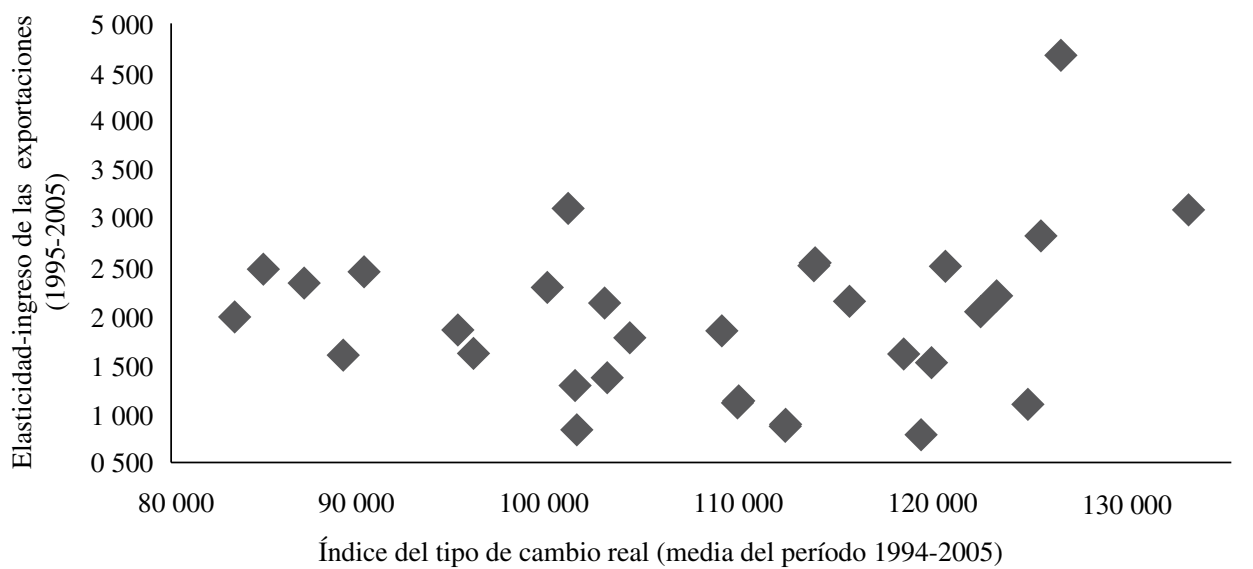

Fuente: elaboración propia sobre la base de datos obtenidos de las Estadísticas financieras internacionales.

CUADRO 9

Países seleccionados: resultados del modelo econométrico de la elasticidad-ingreso de las exportaciones, 1995-2005

\begin{tabular}{lrrrr}
\hline Variable & Coeficiente & Error estándar & Estadístico t & Estadístico p \\
\hline RER & 0,027719 & 0,013431 & 2,063739 & 0,0492 \\
GAP & 0,203742 & 0,112411 & 1,812469 & 0,0815 \\
RERGAP & $-0,001963$ & 0,001019 & $-1,926045$ & 0,0651 \\
Constante & $-0,919443$ & 1,427213 & $-0,644222$ & 0,5251 \\
$\mathrm{R}^{2}$ & 0,226110 & & & \\
Estadístico Durbin-Watson & 2,116491 & & & \\
$\mathrm{P}$ (estadístico F>0) & 0,078975 & & & \\
\hline
\end{tabular}

Fuente: elaboración propia.

Nota: i) Errores estándar consistentes con la heterocedasticidad de White y covarianza; ii) RER: índice del TCR; iii) GAP: relación entre el ingreso per cápita del país en estudio en relación con los Estados Unidos de América, y iv) Dw: prueba de Durbin-Watson para detectar la autocorrelación entre residuales. Véase Asteriou (2006) y Hamilton (1994), donde se ofrece una interpretación exhaustiva de estas pruebas.

y el TCR en los países seleccionados. En los niveles inferiores del TCR parece darse una relación negativa entre ambas variables. No obstante, la relación es positiva en los niveles más elevados del tipo de cambio.

En el cuadro 9 se exponen los resultados del modelo econométrico.

Los índices del TCR y la brecha tecnológica presentan los signos esperados y, estadísticamente, son significativos al $5 \%$ y el $10 \%$, de manera respectiva. Ello significa que la depreciación del TCR aumentará la elasticidad-ingreso de las exportaciones, lo que elevará el crecimiento de las exportaciones locales dada una tasa de engrosamiento de los ingresos mundiales. Se trata de un resultado coherente con la idea de que el grado de especialización productiva de determinada economía depende críticamente del TCR; por ende, existe un vínculo entre el TCR y la tasa de crecimiento a largo plazo.

También es posible apreciar que una reducción de la brecha tecnológica - representada por un aumento del índice de esa brecha - incrementará la elasticidad-ingreso de las exportaciones, lo que entonces confirma la hipótesis de que un mayor nivel tecnológico se vincula a exportaciones de mayor contenido tecnológico, aumentando así la elasticidad-ingreso de las exportaciones del país.

Por último, existe una interacción negativa, pequeña pero estadísticamente significativa, entre el TCR y la brecha tecnológica, lo que corrobora la hipótesis de que el efecto de las variaciones del TCR en la elasticidadingreso de las exportaciones depende del tamaño de la brecha tecnológica. El signo negativo de esta variable en 
las estimaciones de regresión refleja la ponderación de los países desarrollados en la muestra. En estos países la brecha tecnológica es menor, por lo que su competitividad externa les permite mantener monedas valorizadas en relación con las de las economías en desarrollo.

Las pruebas econométricas demuestran que los países más alejados de la "frontera tecnológica" no pueden tomar un tipo de cambio bajo como base para su estrategia de crecimiento. En estos países, la valorización del tipo de cambio eliminará el único modo que tienen de competir con los países desarrollados, a saber, una moneda devaluada. Los países desarrollados pueden compensar el tipo de cambio más bajo con productos que son superiores desde el punto de vista tecnológico.

\section{$\mathrm{V}$}

\section{Conclusiones}

En este artículo se utilizó el modelo de crecimiento impulsado por la demanda para encontrar respuesta a dos interrogantes: i) ¿por qué la tasa de crecimiento de la economía brasileña se desaceleró en las dos últimas décadas del período 1950-1980?, y ii) ¿qué políticas es necesario adoptar para acelerar el crecimiento sostenible de la economía del Brasil?

La respuesta a la primera pregunta se basa directamente en la teoría del crecimiento impulsado por la demanda. Las pruebas econométricas expuestas en la sección III demuestran que el $85 \%$ del crecimiento del PIB real del Brasil en el período 1990-2005 se explica por variables de la demanda agregada, lo que respalda la hipótesis del crecimiento impulsado por la demanda en la economía brasileña. Luego se utilizó la metodología desarrollada por Ledesma y Thirlwall (2002) para demostrar que la tasa natural de crecimiento de la economía brasileña es endógena y se eleva significativamente en los períodos de auge. En consecuencia, no parece haber restricciones de la oferta que impidan un crecimiento económico más rápido.

Desde esta perspectiva, la desaceleración del crecimiento que registró la economía brasileña a principios de la década de 1980 reflejó el agotamiento del patrón de crecimiento de la demanda agregada imperante desde 1964, a saber, una expansión del gasto en bienes durables o de lujo facilitada por la creciente concentración de los ingresos en las clases media y alta. El cuasi estancamiento de la economía brasileña se explica entonces por la
También existe para todos los países que integran la muestra una clara relación positiva entre la elasticidadingreso de las exportaciones y el nivel del TCR. Ello significa que, independientemente del tamaño de la brecha tecnológica, la devaluación del TCR puede elevar la tasa de crecimiento a largo plazo de una economía, al aumentar la elasticidad-ingreso de sus exportaciones e impulsar así el incremento de estas dada una tasa de crecimiento de los ingresos mundiales. Por consiguiente, el TCR constituye una variable fundamental en la estrategia de crecimiento de cualquier país ${ }^{21}$.

21 Véase Gala (2008), que expone un relevamiento del material publicado sobre el TCR y el crecimiento.

actual inexistencia de un patrón constante de expansión de la demanda agregada.

Las pruebas econométricas demostraron, además, que el efecto multiplicador del consumo público corriente es de aproximadamente un $0,40 \%$, por lo que un incremento de un $1 \%$ en el consumo público corriente se traducirá en un aumento de un $0,37 \%$ en el PIB real del Brasil. En el supuesto de una alícuota tributaria media de alrededor del $40 \%$ del PIB, se desprende que un incremento de un $1 \%$ en el consumo público corriente solo elevará la recaudación impositiva en un $0,15 \%$ del PIB. En el marco de la actual crisis fiscal en el Brasil, que importa la combinación de una elevada relación entre la deuda pública y el PIB, altos impuestos y bajos niveles de inversión pública en obras de infraestructura, resulta imposible estimular el crecimiento en la economía acrecentando el consumo público corriente. La única alternativa es adoptar un modelo de crecimiento impulsado por las exportaciones.

¿Qué condiciones deben reunirse para que haya una expansión sólida y constante de las exportaciones en el Brasil o en otras economías capitalistas? Las pruebas econométricas expuestas en la sección III demuestran que los países más alejados de la "frontera tecnológica" no pueden tomar un tipo de cambio bajo como base para su estrategia de crecimiento. En estos países, la valorización del TCR eliminará la única herramienta con que cuentan para competir con los países desarrollados, es decir, una moneda devaluada. En cambio, a los países 
desarrollados se les hace posible compensar el tipo de cambio más bajo con productos que resultan superiores desde el punto de vista tecnológico.

También existe para todos los países que integran la muestra una clara relación positiva entre la elasticidadingreso de las exportaciones y el nivel del TCR. Ello significa que, independientemente del tamaño de la brecha tecnológica, la devaluación del TCR puede elevar la tasa de crecimiento a largo plazo de una economía al aumentar la elasticidad-ingreso de sus exportaciones e impulsar así el incremento de estas en relación con una tasa dada de crecimiento de los ingresos mundiales. Por consiguiente, el tipo de cambio real (TCR) constituye una variable fundamental en la estrategia de crecimiento de cualquier país.

Como corolario de los resultados precedentes, los países en desarrollo, como el Brasil, pueden intentar compensar los efectos que, en materia de competitividad internacional, produce su desventaja tecnológica mediante la devaluación del TCR frente a los tipos de cambio de los países desarrollados. Ello significa que la adopción en el Brasil de un modelo de crecimiento promovido por las exportaciones - condición necesaria para que este país logre tasas elevadas de crecimiento a largo plazo- exige la implementación de una política cambiaria capaz de sostener un TCR devaluado a largo plazo.

\section{Bibliografía}

Asteriou, D. (2006), Applied Econometrics: A Modern Approach Using Eviews and Microfit, Nueva York, Palgrave Macmillan.

Atesoglu, H.S. (2002), "Growth and fluctuations in the USA: a demand oriented approach", The Economics of Demand-Led Growth, M. Setterfield (org.), Aldershot, Edward Elgar.

Barbosa, F.H. (2006), "The contagion effect of public debt on monetary policy: the Brazilian experience", Brazilian Journal of Political Economy, vol. 26, № 2, São Paulo, Centro de Economía Política.

Dornbusch, R., S. Fischer y P. Samuelson (1977), "Comparative advantage, trade and payments in a Ricardian Model with continuum of goods", American Economic Review, vol. 67, $\mathrm{N}^{\circ}$ 5, Nashville, Tennessee, American Economic Association.

Dosi, G., K. Pavitt y L. Soete (1990), The Economics of Technical Change and International Trade, Londres, Macmillan Press.

Engle, R.F. y C.W.J. Granger (1987), "Co-integration and error correction: representation, estimation and testing", Econometrica, vol. 55, $\mathrm{N}^{\circ}$ 2, Nueva York, The Econometric Society.

Fagerberg, J. (1988), "Why growth rates differ", Technical Change and Economic Theory, G. Dosi y otros (orgs.), Londres, Pinter Publishers.

Gala, P. (2008), "Real exchange rate levels and economic development: theoretical analysis and econometric evidence", Cambridge Journal of Economics, vol. 32, $\mathrm{N}^{\circ}$ 2, Oxford, Oxford University Press.

Granger, C.W.J. (1981), "Some properties of time series data and their use in econometric model specification", Journal of Econometrics, vol. 16, $\mathrm{N}^{\circ} 1$, Amsterdam, Elsevier.

Hamilton, J.D. (1994), Time Series Analysis, Princeton, Princeton University Press.

Harrod, R. (1939), "An essay in dynamic theory", The Economic Journal, vol. 49, $\mathrm{N}^{\mathrm{o}}$ 193, Oxford, Blackwell Publishing.

Johansen, S. (1991), "Estimation and hypothesis testing of cointegrating vectors in Gaussian vector autoregressive models", Econometrica, vol. 59, № 6, Nueva York, The Econometric Society.

(1988), "Statistical analysis of cointegration vectors", Journal of Economic Dynamics and Control, vol. 12, No 2-3, Amsterdam, Elsevier.

Johansen, S. y K. Juselius (1990), "Maximum likelihood estimation and inference on cointegration: with applications to the demand for money", Oxford Bulletin of Economics and Statistics, vol. 52, $\mathrm{N}^{\mathrm{o}}$ 2, Oxford, Universidad de Oxford.
Kaldor, N. (1988), "The role of effective demand in the short and long-run growth", The Foundations of Keynesian Analysis, A. Barrére (org.), Londres, Macmillan Press.

(1957), "A model of economic growth", The Economic Journal, vol. 67, № 268, Oxford, Blackwell Publishing.

Kwiatkowski, D. y otros (1992), "Testing the null hypothesis of stationarity against the alternative of a unit root: how sure are we that economic time series have a unit root?", Journal of Econometrics, vol. 54, № 1-3, Amsterdam, Elsevier.

Ledesma, M.L. (2002), "Accumulation, innovation and catchingup: an extended cumulative growth model", Cambridge Journal of Economics, vol. 26, $\mathrm{N}^{\mathrm{o}}$ 2, Oxford, Oxford University Press.

Ledesma, M.L. y A. Thirlwall (2002), "The endogeinity of the natural rate of growth", Cambridge Journal of Economics, vol. 26, $\mathrm{N}^{\circ} 4$, Oxford, Oxford University Press.

Libanio, G. (2009), "Aggregate demand and the endogeinity of the natural rate of growth: evidence from Latin American Countries", Cambridge Journal of Economics, vol. 33, № 5, Oxford, Oxford University Press.

Lucas, R.E. (1988), "On the mechanics of economic development", Journal of Monetary Economics, vol. 22, N ${ }^{\circ}$ 1, Amsterdam, Elsevier.

MacKinnon, J. (1990), "Critical values for cointegration tests", Economics Working Paper Series, No 90-4, San Diego, Universidad de California.

Maddala, G.S. (2001), Introduction to Econometrics, Wiley.

McCombie, J.S.L. y J.R. de Ridder (1984), "The Verdoorn Law controversy: some new empirical evidence using U.S. state data", Oxford Economic Papers, vol. 36, N² 2, Oxford, Oxford University Press.

McCombie, J.S.L. y M. Roberts (2002), "The role of the balance of payments in economic growth", The Economics of Demand-Led Growth, M. Setterfield (org.), Aldershot, Edward Elgar.

Okun, A. (1962), "Potential GNP: its measurement and significance", Proceedings of the American Statistical Association, vol. 7, Alexandria, American Statistical Association.

Park, H.M. (2003), "Testing Normality in SAS, STATA, and SPSS" [en línea] http://pytheas.ucs.indiana.edu.

Park, M.S. (2000), "Autonomous demand and the warranted rate of growth", Contributions to Political Economy, vol. 19, No 1, Oxford, Oxford University Press. 
Romer, D. (1986), "Increasing returns and long-run growth", Journal of Political Economy, vol. 94, No 5, Chicago, University of Chicago Press.

Setterfield, M. (1997), Rapid Growth and Relative Decline, Londres, Macmillan Press.

Solow, R. (1956), "A contribution to the theory of economic growth", Quarterly Journal of Economics, vol. 70, $\mathrm{N}^{\mathrm{o}}$ 1, Oxford, Oxford University Press.
Thirlwall, A. (1969), "Okun's Law and the natural rate of growth", The Southern Economic Journal, vol. 36, № 1, Chattanooga, Southern Economic Association.

(1997), "Reflections on the concept of balance-of-paymentsconstrained growth rates", Journal of Post Keynesian Economics, vol. 19, $\mathrm{N}^{\circ} 3$, M.E. Sharpe Inc.

(2002), The Nature of Economic Growth, Aldershot, Edward Elgar. 